\title{
Heart disease in patients with haemoglobinopathies
}

\author{
Dimitrios Farmakis ${ }^{1,2}$, George Papingiotis ${ }^{1}$ \\ ${ }^{1}$ Cardiac Clinic for Haemoglobinopathies, First Dept. of Internal Medicine, Laiko Hospital, \\ National and Kapodistrian University of Athens Medical School, Athens Greece; ${ }^{2}$ European \\ University Cyprus Medical School, Nicosia, Cyprus
}

Hereditary hemoglobin disorders, also termed haemoglobinopathies, include mainly beta -thalasszemia and sickle cell disease and represent the most common monogenic disorders in human. Cardiac complications are still a leading cause of mortality and morbidity in patients with haemoglobinopathy, although heart disease due to either severe anaemia or iron overload have dramatically reduced in patient populations receiving modern regular therapy and follow-up $(1,2)$. The spectrum of cardiovascular manifestations in haemoglobinopathies is wide and includes ventricular dysfunction, pulmonary hypertension, pericarditis-myocarditis, arrhythmias stroke and thromboembolic events.

The two main determinants of cardiovascular phenotype in haemoglobinopathy patients are the underlying molecular defect responsible for the main disease and the therapy applied for its management (3). In beta-thalassaemia, the basic defect is quantitative and concerns the reduction or total depletion of $\beta$-globin chain synthesis with a relative excess of $\alpha$-globin chains that results in ineffective erythropoiesis. In sickle cell disease, on the other hand, the basic defect is qualitative, as a substitution at sixth amino acid in the $\beta$ chain leads to the synthesis of an abnormal hemoglobin, hemoglobin S, instead of the normal hemoglobin A (4). The common denominator of both molecular defects is the development of chronic haemolytic anaemia, which, particularly when severe and untreated, leads to cardiovascular complications including left and right ventricular dysfunction and pulmonary hypertension (3). In addition, the applied disease-specific therapy may further modify the cardiovascular phenotype by preventing some complications while promoting some others (Figure 1).

Left ventricular dysfunction is the result of several pathophysiological mechanisms, of which high output state and iron overload are the most important ones. Other components of the complex pathophysiology of left ventricular dysfunction include vascular disease (structural and functional) and acute myocarditis. High output state is associated with chronic anaemia and increased percentage of hemoglobin F; significant and un- or mal-treated anaemia results in compensatory bone marrow expansion, while hemoglobin $\mathrm{F}$ binds to oxygen with greater affinity than hemoglobin $A$ and as a result, there is a reduced tissue oxygen delivery (5). Iron overload cardiomyopa-

Correspondence: Dimitrios Farmakis, George Papingiotis, Cardiac Clinic for Haemoglobinopathies, First Dept. of Internal Medicine, Laiko Hospital, National and Kapodistrian University of Athens Medical School, Athens Greece; European University Cyprus Medical School, Nicosia, Cyprus.

This work is licensed under a Creative Commons Attribution 4.0 License (by-nc 4.0).

(C) Copyright D. Farmakis and G. Papingiotis, 2018

Licensee PAGEPress, Italy

Thalassemia Reports 2018; 8:7480

doi:10.4081/thal.2018.7480 thy is primarily seen in transfusion-dependent patients, namely those with thalassemia major or other haemoglobinopathies patients that require regular blood transfusions (e.g., thalassemia intermedia). Repetitive blood transfusions, ineffective erythropoiesis, increased peripheral hemolysis, increased intestinal absorption and lack of proper iron chelation therapy are the main pathogenetic mechanisms associated with the development of iron overload cardiomyopathy $(1,6)$. Iron overload causes further injury to liver and endocrine glands, while it is also believed to affect the immune system causing susceptibility to infections as well as the vascular function, effects that also contribute to the pathophysiology of iron overload cardiomyopathy (7). Acute myocarditis has also been reported as a cause of left ventricular dysfunction and heart failure in thalassaemia major. In addition, beta-thalassaemia and sickle cell disease patients seem to suffer from a complex vasculopathy. This vasculopathy involves both a functional component, characterized by endothelial dysfunction and increased arterial stiffness as well as a structural component concerning an elastic tissue defect similar to the one observed in hereditary pseudoxanthoma elasticum $(8,9)$.

Pulmonary hypertension is a leading cause of cardiovascular morbidity in haemoglobinopathies. In thalassaemia intermedia, pulmonary hypertension has been reported in up to $60 \%$ of patients not receiving blood transfusions, while in sickle cell disease, it is reported in up to $40 \%$ of cases (10). The pathophysiology of pulmonary hypertension is quite complex with several haemoglobinopathy-related factors and other non haemoglobinopathy-related ones holding key roles. Haemolysis, anemia and hypercoagulable state associated with the main disease represent two important factors in the pathogenesis of pulmonary hypertension, leading to increased pulmonary resistance and high cardiac output, while left ventricular dysfunction and pulmonary disease contribute further to the pathophysiology of pulmonary hypertension (10).

The right ventricle also seems to be affected by the above-mentioned mechanisms. Left ventricular dysfunction and pulmonary hypertension are important causes of secondary right ventricular dysfunction, while primary right ventricular cardiomyopathy has also been described in patients with thalassaemia major (11).

Regarding the clinical phenotypes of heart disease, high-output failure and iron overload cardiomyopathy are the two main forms of left ventricular cardiomyopathy. High output failure develops typically in thalassaemia major patients who not regularly transfused and therefore suffer from severe chronic anaemia. Iron overload cardiomyopathy is seen in regularly transfused patients with thalassaemia major that are not properly chelated. Thalassaemia major patients who are properly transfused and chelated have minimal or no heart disease. Pulmonary hypertension is the main cause of heart failure in patients with thalassemia intermedia who are not regularly transfused and is also found with a lower frequency and severity in patients with sickle cell disease (3). The leading cardiac complications in the different forms of haemoglobinopathies are summarized in Table 1.

All the mechanisms previously described set in frame the common pathophysiologic pathways in heart diseases in haemoglo- 
binopathies. The main two determinants of heart disease's phenotype are physician's therapeutic decision and patient's compliance with therapy. These two parameters are the most significant to take into consideration in order to prevent and manage cardiovascular complications in haemoglobinopathies (Figure 1).

Addressing the pathophysiology of haemoglobinopathies is the key to disease-specific therapy including blood transfusions (regular or upon demand) and iron chelation regimens (12). In thalassaemia major patients, regular blood transfusions to maintain a pre-transfusion level of hemoglobin $\geq 10 \mathrm{~g} / \mathrm{dL}$ allows normal growth, prevents anaemia-related complications such as high output state, hypercoagulability and infections and ensures good quality of life $(1,3)$. Iron chelation therapy prevents or manages effectively heart, liver and endocrine disease resulting from iron overload (3). In fact, iron overload cardiomyopathy is nowadays one of the few truly reversible cardiomyopathies. Modern therapy of tha- lassemia major, consisting of regular blood transfusions and iron chelation guided by cardiac magnetic resonance, has actually changed the course of the disease.

Many patients with thalassemia intermedia and those with sickle cell disease are in most cases non-transfusion dependent. Blood transfusions upon demand (in thalassaemia intermedia) or exchange transfusions (in sickle cell disease) in combination with iron chelation and/or hydroxyurea are considered as therapeutic modalities upon the development of disease complications $(13,14)$.

As haemoglobinopathies are demanding clinical entities with multi-system complications, the need for multidisciplinary care in dedicated clinics is of outmost importance. Cardiovascular complications require special care and regular follow-up. In transfusiondependent patients who develop cardiomyopathy and/or iron overload, as documented by cardiac magnetic resonance, regular blood transfusions aiming at haemoglobin $\geq 10 \mathrm{~g} / \mathrm{dL}$ and combined inten-

Table 1. Leading cardiac complications in the different forms of haemoglobinopathies.

\begin{tabular}{ll} 
Haemoglobinopathy & Main heart disease \\
Thalassemia major, not regularly transfused & High output heart failure \\
Thalassemia major, not properly chelated & Iron overload cardiomyopathy \\
\hline Thalassemia major, properly transfused and chelated & Minimal or no heart disease \\
Thalassemia intermedia, not transfused & Pulmonary hypertension \\
\hline Sickle cell anemia & Pulmonary hypertension \\
Sickle thalassemia & Myocardial ischemia \\
& Strokes \\
\hline
\end{tabular}

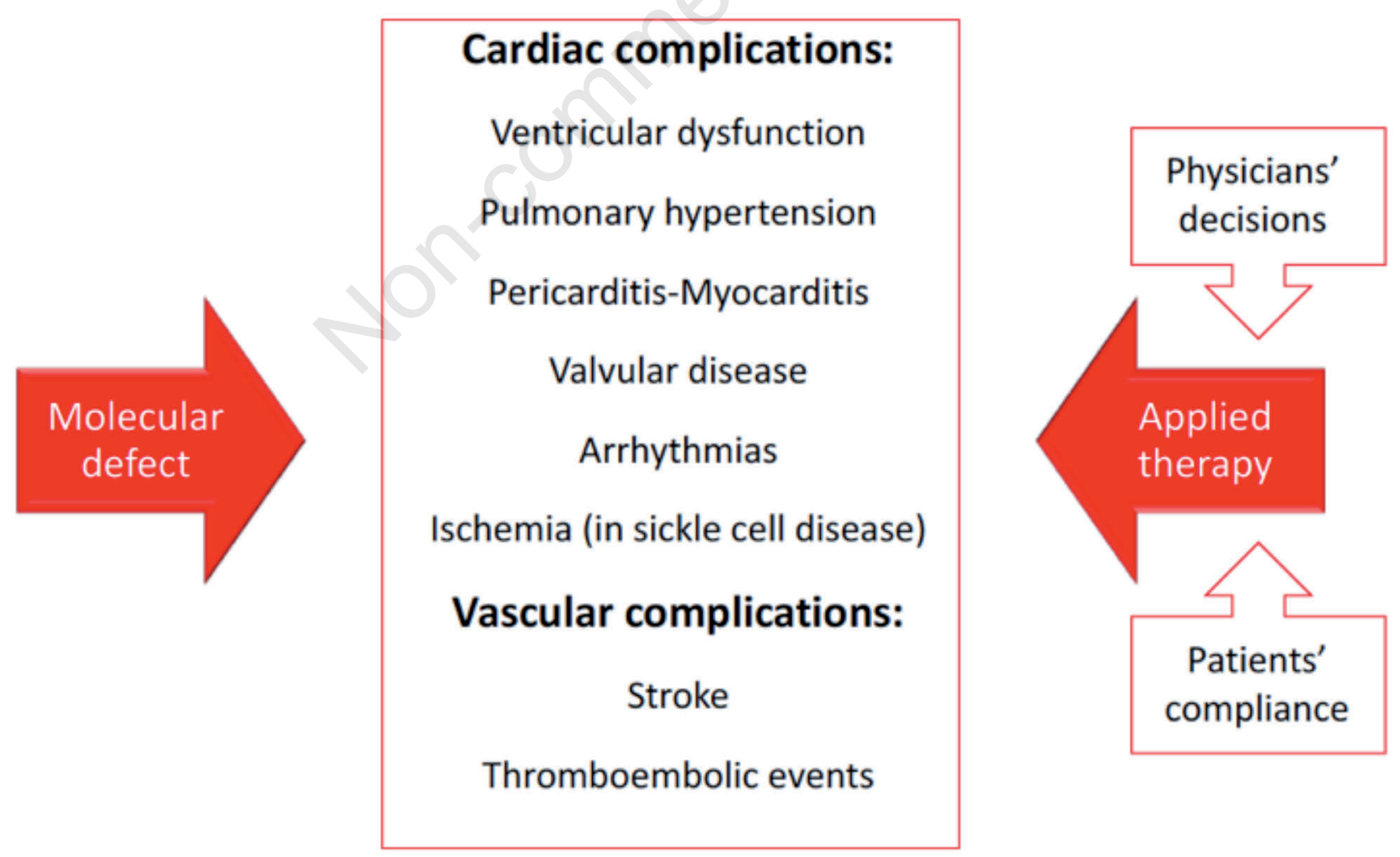

Figure 1. The spectrum of cardiovascular complications seen in patients with haemoglobinopathies is wide and crucially determined by the underlying molecular defect and the applied therapy. 
sive iron chelation plus heart failure therapy (renin-angiotensinaldosterone system inhibitors, $\beta$-blockers, diuretics, device therapy) are key stones in the management of those patients to improve symptoms and prognosis (3). It should be stressed the effect of cardiac magnetic resonance on the survival of those patients; it has been observed a $72 \%$ reduction of mortality due to timely diagnosis of iron overload since early 00 's and a $62 \%$ reduction of allcause mortality (15). A regular follow-up consisting of clinical examination, electrocardiogram, chest radiogram and echocardiography is suggested in all patients. In absence of heart disease, the follow-up is repeated annually and every 6-12 months in case of iron overload (3). Cardiac magnetic resonance is recommended in transfusion dependent patients and repeated according to the clinical course of heart disease. These timeline periods are indicative; on development or change of symptoms and on diagnosis of heart disease, cardiovascular assessment should be repeated according to the proposed algorithms (3).

Finally, treatment of endocrine and other comorbidities in incident haemoglobinopathies and lifestyle modifications that promote cardiovascular health (smoking cessation, regular physical activity, weight control) are also important for the prevention and management of cardiovascular complications.

To summarize, heart disease nowadays is not a leading cause of morbidity and mortality in optimally treated patients, thanks to disease-specific therapy and multidisciplinary management that render cardiovascular complication preventable. Even in the occurrence of heart disease, the early recognition and the effective management that current diagnostic and therapeutic modalities and the knowledge of pathophysiology of the disease offer make heart disease treatable. Regular cardiovascular monitoring in the context of multidisciplinary care and close collaboration with the haemoglobinopathy center are however warranted to ensure the above benefits accomplished by modern therapy and reduce further the morbidity of heart complication in these patients.

\section{References}

1. Kremastinos DT, Farmakis D, Aessopos A, Hahalis G, Hamodraka E, Tsiapras D, Keren A. Beta-thalassemia cardiomyopathy: history, present considerations, and future perspectives. Circ Heart Fail. 2010;3:451-458.

2. Voskaridou E, Ladis V, Kattamis A, Hassapopoulou E, Economou M, Kourakli A, Maragkos K, Kontogianni K, Lafioniatis S, Vrettou E, Koutsouka F, Papadakis A, Mihos A, Eftihiadis E, Farmaki K, Papageorgiou O, Tapaki G, Maili P, Theohari M, Drosou M, Kartasis Z, Aggelaki M, Basileiadi A, Adamopoulos I, Lafiatis I, Galanopoulos A, Xanthopoulidis G, Dimitriadou E, Mprimi A, Stamatopoulou M, Haile ED, Tsironi M, Anastasiadis A, Kalmanti M, Papadopoulou M, Panori E, Dimoxenou P, Tsirka A, Georgakopoulos D, Drandrakis P, Dionisopoulou D, Ntalamaga A, Davros I,
Karagiorga M. Greek Haemoglobinopathies Study Group. A national registry of haemoglobinopathies in Greece: deducted demographics, trends in mortality and affected births. Ann Hematol. 2012;91:1451-8.

3. Farmakis D, Triposkiadis F, Lekakis J, Parissis J. Heart failure in haemoglobinopathies: pathophysiology, clinical phenotypes, and management. Eur J Heart Fail. 2017;19:479-489.

4. Viprakasit V, Origa R. Genetic basis, pathophysiology and diagnosis. In: Cappellini MD, Cohen A, Porter J, Taher A, Viprakasit V. Guidelines for the Management of Transfusion Dependent Thalassaemia. 3rd ed. Thalassemia International Federation.

5. Aessopos A, Farmakis D, Karagiorga M, Voskaridou E, Loutradi A, Hatziliami A, Joussef J, Rombos J, Loukopoulos D. Cardiac involvement in thalassemia intermedia: a multicenter study. Blood. 2001;97:3411-6.

6. Kremastinos DT, Farmakis D. Iron overload cardiomyopathy in clinical practice. Circulation 2011;124:2253-63.

7. Aessopos A, Farmakis D, Andreopoulos A, Tsironi M. Assessment and treatment of cardiac iron overload in thalassemia. Hemoglobin. 2009;33 Suppl 1:S87-92.

8. Aessopos A, Farmakis D, Tsironi M, Diamanti-Kandarakis E, Matzourani M, Fragodimiri C, Hatziliami A, Karagiorga M. Endothelial function and arterial stiffness in sickle-thalassemia patients. Atherosclerosis. 2007;191:427-32.

9. Aessopos A, Farmakis D, Loukopoulos D. Elastic tissue abnormalities resembling pseudoxanthoma elasticum in beta thalassemia and the sickling syndromes. Blood. 2002;99:30-5.

10. Farmakis D, Aessopos A. Pulmonary hypertension associated with hemoglobinopathies: prevalent but overlooked. Circulation. 2011;123:1227-32.

11. Hahalis G, Manolis AS, Apostolopoulos D, Alexopoulos D, Vagenakis AG, Zoumbos NC. Right ventricular cardiomyopathy in beta-thalassemia major. Eur Heart J. 2002;23:147-156.

12. Taher AT, Weatherall DJ, Cappellini MD. Thalassaemia. Lancet. 2018;391:155-167.

13. Walker M, Wood J. Cardiac complications in thalassaemia major. In: Cappellini MD, Cohen A, Porter J, Taher A, Viprakasit V. Guidelines for the Management of Transfusion Dependent Thalassaemia. 3rd ed. Thalassemia International Federation;201. p98-113.

14. Taher AT, Musallam KM, Karimi M, El-Beshlawy A, Belhoul K, Daar S, Saned MS, El-Chafic AH, Fasulo MR, Cappellini MD. Overview on practices in thalassemia intermedia management aiming for lowering complication rates across a region of endemicity: the OPTIMAL CARE study. Blood. 2010;115: 1886-92.

15. Modell B, Khan M, Darlison M, Westwood MA, Ingram D, Pennell DJ. Improved survival of thalassaemia major in the $\mathrm{UK}$ and relation to $\mathrm{T} 2 *$ cardiovascular magnetic resonance. $\mathrm{J}$ Cardiovasc Magn Reson. 2008;10:42. 